\title{
Community Health Workers' Knowledge, Attitudes and Practices about Malaria Prevention in Gicumbi District, Rwanda
}

\author{
Amos Habimana ${ }^{1 *}$, Alexis Harerimana ${ }^{1}$, Domina Asingizwe ${ }^{1}$, Theogene Nyandwi¹, Kato J Njunwa
}

${ }^{1}$ College of Medicine and Health Sciences, University of Rwanda, P.O. Box 3286 Kigali, Rwanda

\begin{abstract}
Background: Community Health Workers (CHWs) have significantly contributed to the decrease of malaria prevalence and related mortality among under five children in Rwanda. This study aimed to explore the knowledge, attitudes and practices of CHWs about malaria prevention in a selected District of Rwanda. Methods: A descriptive method, using self administered questionnaires, was used for data collection from 65 respondents of one randomly selected Sector in Gicumbi District, Rwanda. Results: The majority of the respondents $(89.2 \%, \mathrm{n}=58)$ knew that the mosquito is the vector which transmits malaria to humans, and $(73.8 \%, \mathrm{n}=48) \mathrm{knew}$ correctly the common signs and symptoms of malaria. $(53.8 \%, \mathrm{n}=35)$ were reported to have positive attitude, $(100 \%, \mathrm{n}=65) \mathrm{used}$ mosquito net during night time only, although they are not aware of the specific time of mosquito bite Results on attitudes and practices. There was a statistically significant association between the practices and respondents' age ( $p$ value $=.001$ ), gender ( $p$ value $=.001$ ), respondents' knowledge ( $p$ value $=.001$ ) and respondents' attitudes ( $p$ value $=.001$ ). Conclusion: This study highlights the moderate knowledge on good practices of malaria prevention and management hence improvement with accurate knowledge through appropriate channels is needed, so as CHWs may eventually have good practices in malaria prevention and management.
\end{abstract}

Key words: Community Health Workers, Malaria Prevention

\section{Introduction}

Malaria remains one of the major causes of morbidity and mortality in tropical and subtropical regions of the World (Alonso et al., 2011; WHO, 2014). Over 90\% of the global malaria deaths occur in Sub-Saharan Africa, where the most severe form of the disease prevails, affecting mainly the children aged under five years who bear $78 \%$ of the burden of all malaria-related deaths (WHO, 2014). It has been evidenced that malaria is a major cause of anaemia among pregnant women with, low birth weight, pre-term, foetal and peri-natal mortality, and maternal mortality related to anaemia (Greenwood, Alonso, ter Kuile, Hill, \& Steketee, 2007; Rulisa et al., 2009; Steketee, 2014).

In Rwanda like in many endemic countries, malaria has declined considerably over the last six years, from 55\% in 2002 to 67\% in 2007 (Asingizwe, Rulisa, Asiimwe-kateera, \& Kim, 2015; Karema et al., 2012; O’Meara, Mangeni, Steketee, \& Greenwood, 2010) as a result of diverse control efforts, namely the use of long lasting insecticide treated nets (LLIN), Indoor Residual Spraying, and Artemisinin Combination Therapy (ACT) (White et al., 2014; WHO, 2014). Nevertheless, residual infections in the community still persist and continue to cause morbidity and mortality (Kateera et al., 2015; White et al., 2014). The reported persitance of malaria cases are partly linked to limited community's knowledge, atttitutes and practices towards malaria prevention, and thes remaining issues can be addressed through community health workers' interventions. The future success of malaria prevention and control activities therefore will depend on the sustainablitily of the achievements already made through the strengthening the health care delivery system particularly at community level (Galactionova, Tediosi, de Savigny, Smith, \& Tanner, 2015)

CHWs play an essential role in the task-shifting policy promoted by the World Health Organization and these are considered to be the cornerstone of a global strategy to help Sub-Saharan African countries to achieve the Millennium Development Goals (Druetz, Kadio, Haddad, Kouanda, \& Ridde, 2015). CHWs are usually defined as individuals who received basic health training and work in the community where they live (Druetz et al., 2015).

*Corresponding author, email: habis2012@yahoo.com
It was found that CHWs played major role in the decline of malaria prevalence in Uganda (Mukanga et al., 2012). In Kenya, it was found an increase in reported utilization of CHWs as source of advice/treatment for child fevers from $2 \%$ to $35 \%$ in 2012, accompanied by a decline in careseeking from government facilities (from 67\% to 48\%) and other sources (26\% to $2 \%$ ) including shops (Kisia et al., 2012). CHWs have good knowledge the sense of being valued by the health system and accountability to village members in one region (Zorgho) of Burkinafaso while it was contrary in the other region (Kaya region) where they perceive themselves as being accountable to the health system or, more exactly, to the nurses and sending them a monthly activity report (Druetz et al., 2015). The attitudes of CHWs were found positive, since they expressed their high commitment to attend to patients' needs. A high care taker acceptability was observed, and this may be attributed to the fact that the community members are the ones who choose a person to be trained as a CHW. Another study conducted in Kenya found that CHWs had good knowledge related to malaria transmission, prevention, and management including diagnosis and treatment (Diggle et al., 2014)if not carried out exactly, easily result in the over-or under-diagnosis of malaria. The reliability and accuracy of malaria RDTs, even in extremely challenging health care settings, have made them a staple in malaria control programmes. Using the setting of a pilot introduction of malaria RDTs in Greater Garissa, North Eastern Province, Kenya, this study aims to identify and understand perceptions regarding malaria diagnosis, with a particular focus on RDTs, and treatment among community members and health care workers (HCWs. In Tanzania, the study conducted by (Mubi et al., 2011) it was concluded that the RDTs in the hands of CHWs may safely improve early and well-targeted ACT treatment of malaria patients at community level in Africa.

In 2010, Rwanda Ministry of Health (MoH) has put in place a Community Health Policy and a Information Education and Communication (IEC) teaching guide with the content on Knowledge, Attitudes and Practices to be held by community health workers on malaria prevention in Rwanda. A number of $41,775 \mathrm{CHWs}$ countrywide were trained on integrated community case management including malaria,pneumonia, diahree etc Home Based Management of malaria by the $\mathrm{CHW}$ include performance of Rapid Diagnostic Tests for malaria, fever, and diahrrea. These 
CHWs' interventions resulted in tremendous outcomes whereby $93 \%$ children under five years were being treated at the community level within 24 hours for symptom onset.

The role of (CHW) for managing malaria is considered as one of the key strategies to overcome the human resource gap in the health sector (Condo et al., 2014; Gilmore \& McAuliffe, 2013; Global Health Workforce Alliance and World Health Organization, 2010)evidence is lacking on their effectiveness for specific types of programmes, specifically programmes of a preventive nature. This review reports findings on a systematic review analysing effectiveness of preventive interventions delivered by Community Health Workers for Maternal and Child Health in low- and middle-income countries. Methods: A search strategy was developed according to the Evidence for Policy and Practice Information and Co-ordinating Centrel u2019s (EPPI-Centre. It is on that basis that in 2008 with the introduction of the National Community Health Policy $(\mathrm{MoH}, 2008)$, Rwanda formally launched the implementation of nationwide community case management of malaria by training and deploying community health workers (CHWs) to diagnose febrile cases with rapid diagnostic tests (RDTs) and treating confirmed malaria cases with artemetherlumefantrine (Galactionova et al., 2015; Mugeni et al., 2014)Rwanda introduced integrated community case management (iCCM.

The first CHWs follow up after they received training reveals that $\mathrm{CHWs}$ were motivated to perform their tasks but they needed technical mentoring to master how to fill the forms, how to properly assess, classify, treat the child and counsel the care giver ( $\mathrm{MoH}, 2009)$. Moreover, the study conducted to report on CHW's perceptions of barriers and motivations as well as those of the beneficiaries of the CHWs to provide and access their services in Rwanda, revealed that $\mathrm{CHWs}$ expressed a sincere desire to perform well, particularly because they experienced appreciation by their colleagues and their communities, and perceived themselves as key assets to directly improving their communities' health. While motivated primarily by community recognition and respect, limited skills and opportunity cost impeded their ability to effectively deliver services, as $\mathrm{CHW}$ s carried out their duties in addition to personal income generating activities (Condo et al., 2014).

Considerable efforts have been made in Rwanda to equip community health workers with knowledge about malaria prevention and management. Search for literature, on Africa and particularly for Rwanda indicated that there paucity of studies addressing the knowledge, attitude and practice of health workers for malarial prevention and management. It therefore found to be of interest to find out how comprehensive the health roles are with respect to malaria prevention and management. Thus this study aimed at investigating the KAP of Community health workers as it relates to malaria prevention and management.

\section{Methods}

\section{Study Area and population}

The study was a cross-sectional study conducted in Kaniga sector, which is one of the 21 sectors of Gicumbi District of Northern Province in Rwanda. The sector is divided into five cells (Mulindi, Bugomba, Rukurura, Nyarwambu and Gatoma) and 34 villages. The target population was all 78 CHWs (a binome comprising of a man and a women for general diseases and a women as assistant maternal to follow antenatal care) living in Kaniga sector. This sector was selected puporsively based on its high prevalence of malaria (91 malaria cases) reported in 2012 by the Rwandan Ministry of Helath report to be more prevalent than the rest 20 sectors sharing the borders with Uganda as neighbor country and the one sector of NYAGATARE district which is a holoendemic area of malaria in Rwanda. Furthermore the inhabitants of these sectors are sharing day to day life from one sector to another such as marriage ceremony, accommodation, cultivating, farming, buying and selling their products. So considering these factors the sector was selected.

\section{Sample and sampling technique}

This study used Simple random sampling method to obtain a representative sample of $65 \mathrm{CHWs}$ out of 78 Community Health Workers after computing the sample size with a general rule of the thumb is to always use the largest sample possible

Formula: Sample Size $=n /[1+(n /$ population $)]$ in which $n$ $=Z * Z\left[P(1-P) /\left(D^{*} D\right)\right]$

$\mathbf{P}=$ True proportion of factor in the population, or the expected frequency value $(50 \%)$

D = Acceptable error (0.05\%)

Z.9. The reliability coefficient at 95\% confidence interval

Population size (78 CHWs) of KANIGA Sector

Kaniga sector was divided into two blocks and numbers were assigned accordingly.The first block was composed by 2 cells (Gatoma and Nyarwambu) and the second with 3 cells (Bugomba, Rukurura and Mulindi). A sampling frame list of CHWs from KANIGA sector was used. By simple random sampling method, each concerned participant filled out a questionnaire individually at home until the researcher reached the sample size of $65 \mathrm{CHWs}$, Where it requires the availability and use of a list of all $78 \mathrm{CHWs}$ of Kaniga sector as the study population

\section{Study instrument and data collection}

A self administred questionnaire with closed ended questions with likert scale was used. This questionnaire has been previously used in another study on knowledge, attitudes and practices on malaria prevention and control (Mwanje, 2013), and this was adapted to fit the context. It has 4 parts, namely Socio-demographic, knowledge regarding malaria prevention and management, attitudes towards malaria prevention and management and practices regarding malaria prevention and management. Data collection was carried out in Kinyarwanda as a local language easily understandable by every community health worker. It has been translated in Kinyarwanda and back translated by professional linguists to ensure that the original meaning is retained. To ensure reliability, the questionnaire was pretested with $6 \mathrm{CHWs}$ who are living in Mukarange sector, the neighbor sector of Kaniga sector. Two research assistants were professionals who were trained before they assisted in data collection to ensure uniformity.

\section{Data analysis}

Data were analysed using the Statitistical Package for Social Sciences (SPSS) version 2016 the findings were presented using descriptive statistics tables and percentages and frequencies were reported in univariate analysis, then inferential or analytic statistics. Bivariate anaylsis was performed using chi- square test to measure the association between socio demographic variables and 
malaria prevention practices as well as knowledge, attitudes and malaria prevention practices.. In the analysis, 5\% was the level of significant therefore any $p$ value of $<0.05$ was considered as significant at 95\% confidence interval.

Refering to the semantic differential technique by (Hill, Osgood, Suci, \& Tannenbaum, 1958), attitude is measured by asking a person to rate an issue or topic on a standard set of bipolar adjectives (i.e. with opposite meanings descriptive words). The same methodology was used in the present study.

\section{Ethical considerations}

The study was approved by the Mount Kenya University Ethical Committee prior to its implementation. Written consent were taken from all the respondents before data collection process. The respondents were explained in detail the full description of the research, confidentiality, and voluntary participation. Every received data was treated carefully and privately with no name tag to it. Also other principles of research ethics namely beneficence, respect of human dignity, and justice were upheld in this study.

\section{Results}

\section{Socio-demographic characteristic of respondents}

The response rate was (100\%, $\mathrm{n}=65$ respondnets) (table 1 ). The majority of the participants were female $(57 \%, n=37)$. The mean age of the participants was 39 years with a standard deviation of 7.9 , with the age ranging from 23 to 59 years. The study revealed that the majority of the respondents $(41 \%, n=27)$. were in the age range of 31 - 40 years. Most of the respondents were educated in primary school $(94 \%, \mathrm{n}=61)$. and others in secondary school $(6 \%, n=4)$. and few of them were employed $(2 \%, n=1)$. Out of those who were employed or not, the common income was between 500-1000Rwf/ day $(36 \%, n=23)$. Most of the families had a family size of $1-2$ people $(49 \%, n=32)$. and most of them $(49 \%, n=32)$. had $3-5$ kids below 15 years old. Sixty $(92 \%, n=60)$. households have suffered from malaria disease

\section{Sources of information regarding malaria prevention and management}

The present study reports that every respondent had received some malaria information regarding from different sources. It was found that $(45 \%, \mathrm{n}=29)$.) of the respondents have got information on malaria prevention from health facilities, $(22 \%, n=14)$. received information from radios, $(15 \%, n=10)$. got information from community meetings, while $(16.6 \%, n=11)$. got information from other sources, smaller percentages reported receiving information from magazines T.V, leaflets, newspapers or friends. Those who have mentioned other sources of information , internet was reported among other, either because of their profession or one of their family members had a history of malaria disease.

\section{Knowledge on malaria prevention and management}

The participants answered a total of 10 close ended, multiple choice questions about malaria. Each correct response was given one mark with a total of 10 marks. The mean knowledge score for the respondents was 7.5 out of possible 10 points $(S D=2.15)$. Distribution of knowledge on malaria of the respondents showed that $(15 \%, n=10)$. of subjects had "low knowledge"( $58 \%, n=38)$. of them had "moderate knowledge, while (26\%,n=17). had "high knowledge" All participants $(100 \%, n=65)$ mentioned in the present study that insecticide-treated nets (ITNs) can prevent malaria. the responses for the 10 knowledge questions are summarized in Table 2 . Around $(97 \%, n=63)$. of the respondents knew that malaria can kill people if it is untreated. The questions with the least number of correct answers were $(58.4 \%, n=38)$. and $(61.5 \%, n=40)$. of the respondents reported on ways of malaria prevention and control (item 4 and 10).

Table 1. Socio-demographic characterists of study participants $(\mathrm{N}=65)$

\begin{tabular}{lll}
\hline Variables & Number $(\mathrm{n}=65)$ & Percentage \\
\hline Gender & 37 & 56.9 \\
Female & 28 & 43.1 \\
Male & 65 & 100.0 \\
Total & & \\
Age group (years) & 9 & 13.8 \\
21-30 years old & 27 & 41.5 \\
31-40 years old & 24 & 36.9 \\
41-50 years old & 5 & 7.7 \\
$51-60$ years old & &
\end{tabular}

Mean $=39.2 \mathrm{Sd}=8.45$ Minimum $=23$ Maximum $=59$

Marital status

65

100.0

Education level

Primary

61

93.8

Secondary

6.2

Total

4

100.0

Professional qualification

Education

1.5

None

98.5

Total

100.0

Employment status

Employed by others

Self employed

1.5

Unemployed

49.2

Total

49.2

32

100.0

Training as a $\mathrm{CHW}$

Yes

96.9

No

63

3.1

Total

2

100.0

Income(Rwf/day)

None

36.9

500-1000Rwf

24

55.4

1000-1500Rwf

36

3.1

Above 1501Rwf

2

4.6

Total

3

100.0 
Table 2. Knowledge on malaria prevention and management $(n=65)$.

\begin{tabular}{lll}
\hline Level & Number (N=65) & Percentage \\
\hline 1. Which vector can transmit malaria to humans? & 58 & 89.2 \\
2. Malaria can be transmitted to humans by? & 19 & 29.2 \\
3. Do you think malaria can kill someone if it's untreated? & 63 & 96.9 \\
4. Which of these are ways to prevent and control malaria? & 40 & 61.5 \\
5. When do malaria mosquitoes feed? & 20 & 30.7 \\
6. What do you think are the most common signs and symptoms in malaria infection? & 48 & 73.8 \\
7. If you or a member of the household were to present with signs and symptoms of malaria, & 62 & 95.38 \\
where would you seek treatment & 60 & 92.3 \\
8. How soon after suspecting malaria would you seek treatment? & 50 & 76.9 \\
9. Do you think you have enough information on malaria? & 38 & 58.4 \\
10. What information have you got about malaria? & & \\
\hline
\end{tabular}

\section{Attitude on malaria prevention and management}

When both positive and negative statements are scored with the right answer scoring 4 points and the wrong answer 1 point following the likert scale, an overall attitude score is determined for each respondent by adding up the scores across the 12 attitude questions. Participants answered a total of 12 questions which had a total score of 60 . There were (53.8, $\%, n=35)$. of respondents who had "positive attitude", (33.8\%,n=22). of them had "neutral attitude", while (12.3\%,n=8). had "negative attitude".

Table 3. Attitude towards malaria prevention and management (ranked by strongly Agree/Agree and Strongly Disagree/Disagree)

\begin{tabular}{|c|c|c|}
\hline Statements & $\begin{array}{l}\text { Strongly agree/ } \\
\text { Agree (\%) }\end{array}$ & $\begin{array}{l}\text { Strongly disagree/ } \\
\text { Disagree (\%) }\end{array}$ \\
\hline 1.Malaria is a disease that cannot be prevented. & 10.0 & 90.0 \\
\hline 2.eliminating breeding places is a responsibility of public health staff and $\mathrm{CHWs.}$ & 85.3 & 14.7 \\
\hline $\begin{array}{l}\text { 3.Only method of managing and preventing malaria disease is to combat the vector } \\
\text { mosquitoes. }\end{array}$ & 96.7 & 3.3 \\
\hline 4.Only spraying is enough to prevent mosquito no need for other ways. & 34.5 & 65.5 \\
\hline 5. Everybody has the chance to be infected with malaria disease. & 92.3 & 7.7 \\
\hline 6.Person who once got malaria disease cannot get malaria disease again. & 6.8 & 93.2 \\
\hline 7.It is impossible to recover completely from malaria disease. & 18.9 & 81.1 \\
\hline 8. Elimination of larval breeding sources is a waste of time and very complicated. & 21.4 & 78.6 \\
\hline $\begin{array}{l}\text { 9. Restricting and checking availability of potential breeding habits should be } \\
\text { conducted day by day. }\end{array}$ & 94.1 & 5.9 \\
\hline 10.Strong and healthy person will not get malaria disease. & 10.2 & 89.8 \\
\hline 11.Sleeping in mosquito net doesn't give the guarantee of malaria prevention. & 22.7 & 77.3 \\
\hline 12.You are one of the important people in preventing malaria transmission. & 88.1 & 11.9 \\
\hline
\end{tabular}




\section{Practice on malaria prevention and management}

Respondents answered nine questions related to the practices of prevention and management of malaria in the study and these are summarised in table 4.

Table 4. Participants practices: malaria prevention and management $(n=65)$

\begin{tabular}{llc}
\hline Items & Number & Percentage \\
\hline 1.Sleeping in mosquito net & 65 & 100.0 \\
2.Visiting the health centre when h/s falls sick & 60 & 92.3 \\
3.Cleaning stagnant water near your house & 58 & 89.2 \\
4.Cleaning/cutting bushes around your house & 61 & 93.8 \\
5.The other members of the household sleep in bed nets & 65 & 100.0 \\
6. Checking for holes /repair bed nets & 45 & 69.2 \\
7. Receiving visits from the heath centre team & 54 & 83.0 \\
8.Using mosquito repellent coils on your house & 2 & 3.0 \\
9. using anti- mosquito spray in your house & 8 & 12.3 \\
\hline
\end{tabular}

All respondents $(100 \%, \mathrm{n}=65)$. had their members of the household sleeping in mosquito nets as a very good practice. The malaria practices with less frequently done by respondents were $(3.0 \%, n=2)$. and $(12.3 \%, n=8)$., for the question regarding the use of mosquito repellent coils and anti- mosquito spray.

Table 5. Association of malaria prevention and management practices with demographic variables, knowledge and attitudes

\begin{tabular}{|c|c|c|c|c|}
\hline \multirow[t]{2}{*}{ Variables } & \multicolumn{4}{|c|}{ Malaria prevention and management practices } \\
\hline & $\begin{array}{l}\text { Good Practice. } \\
\text { Number(\%) }\end{array}$ & $\begin{array}{l}\text { Fair Practice. } \\
\text { Number(\%) }\end{array}$ & $\begin{array}{l}\text { Poor practice. } \\
\text { Number(\%) }\end{array}$ & $P$ value \\
\hline \multicolumn{5}{|l|}{ Age } \\
\hline 21-30 years old & $9(13.8)$ & $O(.0)$ & $0(.0)$ & 0.001 \\
\hline $31-40$ years old & $27(41.5)$ & $O(.0)$ & $O(.0)$ & \\
\hline $41-50$ years old & $8(12.3)$ & 16(24.6) & $1(1.5)$ & \\
\hline $51-60$ years old & $O(.0)$ & $\mathrm{O}(.0)$ & $4(6.1)$ & \\
\hline \multicolumn{5}{|l|}{ Gender } \\
\hline Male & 28(43.1) & $\mathrm{O}(.0)$ & $O(.0)$ & 0.001 \\
\hline Female & $16(24.6)$ & $16(24.6)$ & $5(7.7)$ & \\
\hline \multicolumn{5}{|l|}{ Income level } \\
\hline None & 18(27.7) & $6(9.2)$ & $\mathrm{O}(.0)$ & 0.366 \\
\hline 500-1000Rwf & 22(33.8) & $9(13.8)$ & $5(7.7)$ & \\
\hline 1000-1500Rwf & $1(1.5)$ & $1(1.5)$ & $O(.0)$ & \\
\hline Above 1501Rwf & $3(4.6)$ & $O(.0)$ & $O(.0)$ & \\
\hline \multicolumn{5}{|l|}{ Education level } \\
\hline Primary & $40(61.5)$ & $16(24.6)$ & $5(7.7)$ & 0.362 \\
\hline Secondary & $4(6.1)$ & $O(.0)$ & $O(.0)$ & \\
\hline \multicolumn{5}{|l|}{ Knowledge level } \\
\hline High (8-10 scores) & $17(26.2)$ & $O(.0)$ & $O(.0)$ & 0.001 \\
\hline Moderate(5-7scores) & $27(41.5)$ & 11(16.9) & $\mathrm{O}(.0)$ & \\
\hline Low(0-4 scores) & $O(.0)$ & $5(7.7)$ & $5(7.7)$ & \\
\hline \multicolumn{5}{|l|}{ Attitude level } \\
\hline Positive (scores =>53) & $35(53.8)$ & $\mathrm{O}(.0)$ & $\mathrm{O}(.0)$ & 0.001 \\
\hline Neutral (scores 45-52) & 9(13.8) & 13(20.0) & $\mathrm{O}(.0)$ & \\
\hline Negative (scores<=44) & $\mathrm{O}(.0 \%)$ & $3(4.6 \%)$ & $5(7.7 \%)$ & \\
\hline
\end{tabular}

The attitude had an association between the levels of practice behaviors against malaria prevention and management among the respondents (table 5). Knowledge, Attitudes and Practices regarding malaria prevention and management were also treated as continuous variables and correlation coefficients were computed. Knowledge about malaria prevention 
and management had significant positive correlation with practices of malaria preventive and management behavior ( $P$ value <.001), meaning people who have high knowledge on malaria will have good practices against malaria prevention and management. In comparison, attitude showed also a correlation with level of practices against malaria prevention and management ( $P$ value $<.001$ ), as shown in Table 5. Hereafter, there was uniformity between chi-square testing and correlation analysis concerning the associations of knowledge and attitude with practice. This inferred that there was no major bias due to the preference of cut off points for classifying the knowledge and attitude.

\section{Discussion}

\section{Knowledge about malaria prevention and management}

The mean survey score was found to be 7.5 from a possible 10 points with a standard deviation of 2.15. The reported score shows that the CHWs have good knowledge of malaria prevention and management, whereby more than a half of the participants $(59 \%, n=38)$ in this study had moderate knowledge related to malaria prevention and management, however a significant number (15.4 $\%, n=10$ ) had low level of knowledge despite the fact that $(100 \%, n=65)$. of the respondents had received information regarding malaria. Information regarding malaria prevention and management were reported to be from were diverse sources where radio is the main source $(62.9 \%, n=41)$., followed by the health centre $(23.6 \%, n=15)$. and the village health team members $(17.9 \%, n=12)$. were the most common sources of information amongst those that had received information about malaria as it is also documented in other studies (Mwanja, 2013). The present study showed that the health centre is the main source $(45 \%, n=29)$., followed by radio $(22 \%, n=14)$. and the village health team members $(16.6 \%, n=11)$. were the most common sources of information. Knowledge about malaria prevention was reportedly mentioned that study participants knew that insecticide-treated nets (ITNs) can prevent malaria.

The results of this study showed that the demographic data were not correlated with level of practice scores except for age and gender ( $p$ value=0.001). Therefore, there is a weak association between demographic data and the level of practice excluding the variable of age and gender. This is in contrast with the study by (Haque et al., 2009)malaria cases have increased steadily. Malaria persists as a major health problem in the thirteen south-eastern and north-eastern districts of Bangladesh. At present the national malaria control program, largely supported by the Global Fund for AIDS, Tuberculosis and Malaria (GFATM which indicates that social demographic characteristics, such as marital status, education level etc..., greatly influence performance of $\mathrm{CHWs}$. The study revealed that $\mathrm{CHWs}$ who were married and with good educational background perform better since they are respected by the community.

In the same perspective, the Nigerian study showed that CHWs with higher education were able to train and had a better understanding of skills needed to diagnose and treat common illnesses(Bagonza, Kibira, \& Rutebemberwa, 2014).While the benefits of having basic education are noticeable, evidence from Peru, showed that CHWs with higher education had opportunities for alternative employment and tended to migrate from one job to another. Nevertheless a study in western Uganda showed that age, sex and educational level were not significantly associated with the ability of CHWs to diagnose pneumonia (Bagonza et al., 2014).

The lack of association in most of sociodemographic variables is linked to the limited study sample in the present study compared to the reported literature. On the other hand, the confounding factors may play a big role.

\section{Attitude towards malaria prevention and management}

Attitude towards malaria prevention and management was reported to be positive, where the mean survey score for attitude about malaria prevention was 51.2 from a possible 60 points with a standard deviation of 7.2. In addition, more than a half of the study participants $(53.8 \%, n=35)$. were repored to have positive attitude. On the other hand, the present study revealed out some negative attitudes towards malaria prevention were among the respondents whereby $(34.5 \%, n=23)$. of the respondents felt that only spraying is enough to prevent mosquito no need for other ways.

Activities towards malaria prevention and management an other diseases undertaken by $\mathrm{CHW}$ are coupled with a number of other activities which require supportive supervision, clearly defined roles with specific tasks, locally relevant incentive systems, acknowledgment, training, community and policy support, and strong leadership. They include but not limited to circulating e- health messages, support immunization campaigns, facilitate referrals to health centers or administer treatments for the most widespread diseases, specifically malaria, pneumonia. The aforementioned responsibilities can play a role in the length of time a CHW serves thus affecting their performance and these are in line with documented literature by (Bagonza et al., 2014)

\section{Practices towards malaria prevention and management}

This study reported a significant association with attitude and practices of malaria prevention and management $(P<0.001)$. Regarding practices towards malaria prevention, the majority of the respondents $(41 \%, n=27)$.used mosquito coils or mosquito mats during night time only and $(28 \%, n=18)$. of the respondents did not use anything. Thirty percent used mosquito net during day and night time and only one percent used it during day time. It is in the vein with (Singh, Musa, Singh, \& Ebere, 2014)study which shows misconceptions about malaria transmission and its cause still exist whereby knowledge about preventive measures is translated into improved practices. There is a need for targeted educational programs to increase the communities' efforts to develop desirable attitude and practices regarding malaria and their participation for malaria control.. The reported data are contrary to the study of Mwanje (2013) on Knowledge, Attitude and Practices (KAP) on malaria among selected rural communities in Uganda, a case study of Nsaabwa village, Mukono district which found out that there was no significant association seen between attitude and preventive practice on malaria. May be these people believe and might not appreciate the importance of other malaria preventive measures. The respondents believe that elimination of larval breeding sources is a waste of time and very complicated. The limited preventive measures can negative affect the target by the Rwandan Ministry of Health, to eliminate malaria and its transmission. Therefore,here is a need for a determined effort of collaboration with various public and private organizations in ensuring malaria prevention and management. 


\section{Areas of high level of knowledge}

The highest average percentage scored by the respondents was the question on whether malaria can kill people if it's untreated. The responses indicated that $96.9 \%$ were aware that malaria can kill people if it's untreated. The respondents of this study had a good knowledge (95.38 $\%, n=62$ ) regarding where they would seek treatment when sick Another area in which the respondents scored well is the question about how soon after suspecting malaria they would seek treatment. An average of $(92.3 \%, n=60)$. respondents who participated in the survey got the answers correct to this question as shown in this study.

Knowledge was significantly associated with practice $(P$ value $<0.001)$. The reported evidence implies that improved knowledge regarding malaria positively affect practice of malaria prevention and management. Measures against mosquitoes are probably only used when people experience a mosquito nuisance. Few of the respondents did not see malaria as a threat to their community; therefore proper prevention and management programs need to be developed to make the CHWs more aware which will then motivate behavior change. Consequently this increased awareness will in turn might bring further benefits to the community and this highly beneficial process will continue overtime. Closing the gap between knowledge and practice will continue to be a vital challenge for malaria control, as well as targets for reduction of mosquitoes.It is was reported that CHWs' performance on malaria symptoms was similar in the intervention and control arms on: overall knowledge, eliciting signs and symptoms, and prescribing .More children treated by $\mathrm{CHWs}$ received prompt and appropriate malaria treatment compared to other health providers (37\% vs 9\%, $p<0.001$ ) (Kalyango et al., 2013).

\section{Areas of knowledge deficit}

The very important area that the respondents lacked knowledge was linked to the question on when do malaria mosquitoes feed. In this study, only $(30 \%, n=20)$. of the respondents answered it correctly which means the rest $(70 \%, n=46)$. of the respondents had no idea regarding it. This is an important area which needs to be emphasized more during the prevention programs. ( $29 \%, n=45)$. of the respondents felt that malaria can be transmitted from one person to another. Lack of knowledge regarding malaria that can occur throughout the year would make people lack of awareness on preventive and management behavior of malaria.

Among the participants, knowledge had significant positive association with practice of malaria prevention. This result was consistent with the study of (Koenraadt et al., 2006) who found out in the study that there is a direct link between knowledge on malaria prevention and practices, whereas measures against adult mosquitoes are used only when people experience a mosquito nuisance problem. It was consistent with another study which was done by (van Benthem, Khantikul, Panart, Somboon, \& Oskam, 2006)and to compare the use of prevention measures between people with and without a knowledge of malaria. An epidemiological survey was conducted in January 2002 among 857 persons living in four sites in northern Thailand. Of the 857 persons, 53\% had a knowledge of malaria, ranging from 38\% and 51\% in non-endemic to $76 \%$ and $77 \%$ in endemic sites. Headache $189 \%$, who found out that people with knowledge of malaria reported a significantly higher use of prevention measures than people without knowledge of malaria. It's also supported by studies which proved that improved community knowledge of malaria and its source of transmission promote preventive and personal protection practices amongst the affected community (Ahorlu, Koram, De Savigny, \& Weiss, 2006)

Future CHWs'training on malaria prevention and management should put emphasis on those areas where they have a knowledge deficit about malaria as knowledge is positively associated with practice. Therefore, if they had better knowledge about malaria then they would also be likely to have a better practice regarding malaria prevention.

\section{Conclusion}

The present study shows that Community Health Workers are aware of the good practices of malaria prevention and management however, limited knowledge was highlighted in the areas of using mosquito repellent coils and antimosquito spray in houses in addition to the use of mosquito bednets alone. Further training, awareness and community health workers empowerment activities are required to bridge that gap.

\section{Limitations of the study}

This study is limited in scope and methodology. The sample size was small and this can affect external validity. The method using a cross-sectional descriptive study design to describe the knowledge, attitude and practices of $\mathrm{CHWs}$ in relation to malaria prevention and management do not attempt to generalize the findings to populations outside the study participants. Therefore, findings of this study could not be generalized beyond the participants of the study.

Another limitation of this study as it has used a structured questionnaire to collect data from the survey population. This can limit the responses that the people could choose from the questionnaire and do not have the capacity for in- depth answers. As malaria is a vast and very important topic, there some aspects were not been included in the questionnaire leaving many areas of knowledge not explored. This article also reports the findings from different studies those were undertaken long time ago, hence some of the issues observed might have changed currently. Therefore, the conclusion presented under should be taken carefully. Yet, the study still present positive evidence justifying the needs for better management of the disease to be done regularly as CHWs'training on malaria prevention and management is necessary to address the knowledge gap revealed in the study.

\section{Acknowledgements}

The present study was made possible with financial assistance from the parents.

We appreciate local authorities and all the respondents in KANIGA Sector for their cooperation. Data storage and household data entry was assisted by Dr. Juma Joseph (Ph.D) who provided also excellent official administrative support.

\section{Declaration}

The authors declare no conflict of interest in relation to presentation of this manuscript. 


\section{Authors' contributions}

HA: Conceived research idea, study protocol design, implementation and manuscript writing.

HA: Participated in research protocol design

AD: Participated in manuscript writing

NT: Participated in manuscript writing

KN: Participated in study protocol design, implementation and manuscript writing

\section{References}

Ahorlu, C. K., Koram, K. A., Ahorlu, C., De Savigny, D., \& Weiss, M. G. (2006). Socio-cultural determinants of treatment delay for childhood malaria in southern Ghana. Tropical Medicine and International Health, 11(7), 1022-1031. doi:10.1111/j.13653156.2006.01660.x

Alonso, P. L., Brown, G., Arevalo-Herrera, M., Binka, F., Chitnis, C., Collins, F., Tanner, M. (2011). A research agenda to underpin malaria eradication. PLoS Medicine, 8(1), e1000406. doi:10.1371/journal. pmed.1000406

Asingizwe, D., Rulisa, S., Asiimwe-kateera, B., \& Kim, M. J. (2015). Malaria elimination practices in rural community residents in Rwanda: A cross sectional study. Rwanda Journal Series F: Medicine and Health Sciences, 2(1), 53-59. doi:http://dx.doi.org/10.4314/ rj.v2i1.7F

Bagonza, J., Kibira, S. P. S., \& Rutebemberwa, E. (2014). Performance of community health workers managing malaria, pneumonia and diarrhoea under the community case management programme in central Uganda: a cross sectional study. Malaria Journal, 13(1), 367. doi:10.1186/1475-2875-13-367

Chanda, P., Hamainza, B., Moonga, H. B., Chalwe, V., \& Pagnoni, F. (2011). Community case management of malaria using ACT and RDT in two districts in Zambia: achieving high adherence to test results using community health workers. Malaria Journal, 10(1), 158. doi:10.1186/1475-2875-10-158 [doi]

Condo, J., Mugeni, C., Naughton, B., Hall, K., Tuazon, M. A., Omwega, A., Binagwaho, A. (2014). Rwanda's evolving community health worker system: a qualitative assessment of client and provider perspectives. Human Resources for Health, 12(1), 71. doi:10.1186/1478-4491-12-71

Diggle, E., Asgary, R., Gore-Langton, G., Nahashon, E., Mungai, J., Harrison, R., Allan, R. (2014). Perceptions of malaria and acceptance of rapid diagnostic tests and related treatment practises among community members and health care providers in Greater Garissa, North Eastern Province, Kenya. Malaria Journal, 13, 1-12. doi:10.1186/1475-2875-13-502

Druetz, T., Kadio, K., Haddad, S., Kouanda, S., \& Ridde, V. (2015). Do community health workers perceive mechanisms associated with the success of community case management of malaria? A qualitative study from Burkina Faso. Social Science \& Medicine (1982), 124, 232-40. doi:10.1016/j. socscimed.2014.11.053

Galactionova, K., Tediosi, F., de Savigny, D., Smith, T., \& Tanner, M. (2015). Effective coverage and systems effectiveness for malaria case management in subSaharan African countries. PloS One, 10(5), e0127818. doi:10.1371/journal.pone.0127818

Gilmore, B., \& McAuliffe, E. (2013). Effectiveness of community health workers delivering preventive interventions for maternal and child health in lowand middle-income countries: a systematic review. BMC Public Health, 13(1), 847. doi:10.1186/14712458-13-847

Global Health Workforce Alliance and World Health Organization. (2010). Global Experience of Community Health Workers for Delivery of Health Related Millennium Development Goals: A Systematic Review, Country Case Studies, and Recommendations for Integration into National Health Systems. Community Health.

Greenwood, B., Alonso, P., ter Kuile, F. O., Hill, J., \& Steketee, R. W. (2007). Malaria in pregnancy: priorities for research. Lancet Infectious Diseases. doi:10.1016/ s1473-3099(07)70028-2

Haque, U., Ahmed, S. M., Hossain, S., Huda, M., Hossain, A., Alam, M. S., Haque, R. (2009). Malaria prevalence in endemic districts of Bangladesh. PLOS ONE, 4(8), 1-9. doi:10.1371/journal.pone.0006737

Hill, R. J., Osgood, C. E., Suci, G. J., \& Tannenbaum, P. H. (1958). The Measurement of Meaning. American Sociological Review, 23(2), 227. doi:10.2307/2089024

Kalyango, J. N., Alfven, T., Peterson, S., Mugenyi, K., Karamagi, C., \& Rutebemberwa, E. (2013). Integrated community case management of malaria and pneumonia increases prompt and appropriate treatment for pneumonia symptoms in children under five years in Eastern Uganda. Malaria Journal, 12(1), 340. doi:10.1186/1475-2875-12-340

Karema, C., Aregawi, M. W., Rukundo, A., Kabayiza, A., Mulindahabi, M., Fall, I. S., Binagwaho, A. (2012). Trends in malaria cases, hospital admissions and deaths following scale-up of anti-malarial interventions, 2000-2010, Rwanda. Malaria Journal, 11, 236. doi:10.1186/1475-2875-11-236

Kateera, F., Mens, P. F., Hakizimana, E., Ingabire, C. M., Muragijemariya, L., Karinda, P., van Vugt, M. (2015). Malaria parasite carriage and risk determinants in a rural population: a malariometric survey in Rwanda. Malaria Journal, 14(1), 16. doi:10.1186/s12936-0140534-x

Kisia, J., Nelima, F., Otieno, D., Kiilu, K., Emmanuel, W., Sohani, S., Akhwale, W. (2012). Factors associated with utilization of community health workers in improving access to malaria treatment among children in Kenya. Malaria Journal, 11(1), 248. doi:10.1186/1475-2875-11-248

Koenraadt, C. J. M., Tuiten, W., Sithiprasasna, R., Kijchalao, U., Jones, J. W., \& Scott, T. W. (2006). Dengue knowledge and practices and their impact on Aedes aegypti populations in Kamphaeng Phet, Thailand. 
American Journal of Tropical Medicine and Hygiene, 74(4), 692-700. doi:74/4/692 [pii]

$\mathrm{MoH}$. (2008). National Community Health Policy. Wikischolars. Columbia. Edu. KIGALI: MINISTRY OF HEALTH RWANDA.

MoH.(2009). Annual Report July 2010 - June 2011. Edu. KIGALI: MINISTRY OF HEALTH RWANDA.

MoH.(2010). Annual Report July 2010 - June 2011. Edu. KIGALI: MINISTRY OF HEALTH RWANDA.

Mubi, M., Janson, A., Warsame, M., Mårtensson, A., Källander, K., Petzold, M. G., Björkman, A. (2011). Malaria rapid testing by community health workers is effective and safe for targeting malaria treatment: Randomised cross-over trial in Tanzania. PLoS ONE, 6(7). doi:10.1371/journal.pone.0019753

Mugeni, C., Levine, A. C., Munyaneza, R. M., Mulindahabi,

E., Cockrell, H. C., Glavis-Bloom, J., Binagwaho, A. (2014). Nationwide implementation of integrated community case management of childhood illness in Rwanda. Global Health, Science and Practice, 2(3), 328-41. doi:10.9745/GHSP-D-14-00080

Mukanga, D., Tibenderana, J. K., Peterson, S., Pariyo, G. W., Kiguli, J., Waiswa, P., Källander, K. (2012). Access, acceptability and utilization of community health workers using diagnostics for case management of fever in Ugandan children: a cross-sectional study. Malaria Journal, 11, 121. doi:10.1186/1475-287511-121 [doi]

Mwanje, L. (2013). Knowledge, Attitudes and Practices on Malaria Prevention and Control in Uganda Knowledge, Attitudes and Practices on Malaria Prevention and Control in Uganda.

O'Meara, W. P., Mangeni, J. N., Steketee, R., \& Greenwood, B. (2010). Changes in the burden of malaria in sub-Saharan Africa. The Lancet Infectious Diseases. doi:10.1016/S1473-3099(10)70096-7

Perry, H. B., Zulliger, R., \& Rogers, M. M. (2014). Community Health Workers in Low-, Middle-, and High-Income Countries: An Overview of Their History, Recent Evolution, and Current Effectiveness. Annual Review of Public Health, 35(1), 399-421. doi:10.1146/ annurev-publhealth-032013-182354

Rulisa, S., Mens, P. F., Karema, C., Schallig, H. D. F. H., Kaligirwa, N., Vyankandondera, J., \& de Vries, P. J. (2009). Malaria has no effect on birth weight in Rwanda. Malaria Journal, 8, 194. doi:10.1186/14752875-8-194

Singh, R., Musa, J., Singh, S., \& Ebere, U. V. (2014). Knowledge, attitude and practices on malaria among the rural communities in aliero, northern Nigeria. Journal of Family Medicine and Primary Care, 3(1), 3944. doi:10.4103/2249-4863.130271

Sirima, S. B., Konate, A., Tiono, A. B., Convelbo, N., Cousens, S., \& Pagnoni, F. (2003). Early treatment of childhood fevers with pre-packaged antimalarial drugs in the home reduces severe malaria morbidity in Burkina Faso. Tropical Medicine and International Health, 8(2), 133-139. doi:10.1046/j.1365-3156.2003.00997.x
Steketee, R. W. (2014). Malaria Prevention during Pregnancy-ls There a Next Step Forward? PLoS Medicine, 11(9), e1001734. doi:10.1371/journal. pmed.1001734

van Benthem, B. H. B., Khantikul, N., Panart, K., Somboon, P., \& Oskam, L. (2006). Knowledge and use of preventive measures against malaria in endemic and non-endemic villages in northern Thailand. Southeast Asian Journal of Tropical Medicine \& Public Health, 37(2), 243-249.

White, N. J., Pukrittayakamee, S., Hien, T. T., Faiz, M. A., Mokuolu, O. a, \& Dondorp, A. M. (2014). Malaria. Lancet, 383, 723-35. doi:10.1016/S01406736(13)60024-0

WHO. (2014, February). World Malaria Report 2014. Geneva: WHO. doi:10.1007/s00108-013-3390-9 